\title{
OBSERVATIONS REGARDING ON EXISTING ENTOMOFAUNA FROM WALNUT ORCHARDS
}

\author{
Ionel Perju ${ }^{1}$, Mihai Tălmaciu ${ }^{1}$, Nela Tălmaciu ${ }^{1}$, Monica Herea ${ }^{1, *}$ \\ ${ }^{1}$ University of Life Science „Ion Ionescu de la Brad” from Iasi, Iași, Romania
}

Current Trends in

Natural Sciences

\begin{abstract}
The observations were made during the two years, 2019 and 2020 in a plantation belonging walnut fruit from Iasi County in the chemical treated stationary, in both years. The material collection was done with traps type Barber from May until August at intervals between 10 and 20 days. Collection of 2019 was made on the following dates: 20.05, 30.05, 15.06, 5.07, 25.07, 10.08 and 23.08. In total 2019 were collected 265 samples belonging to 22 species (taxa). Species (taxa) with the largest number of samples collected were Dermestes laniarius L. 42 samples Heteroptera (bedbugs) with 38 samples, and Polydrosus sericeus Schall. 26 samples. In 2020 collection of the biological material was made on the following dates: 10.05, 22.05,18.06, 06.07, 29.07, 15.08, 05.09. In total in 2020 were collected 744 samples belonging to 41 species (taxa) with the highest number of samples were colected: Hymenoptera (bees) with 92 saples, Orthoptera (locusts) with 87 samples, Lepidoptera (larvae) with 37samplres, Araneida (spiders) with 33 samples and Homoptera (cycads) with 31 samples.
\end{abstract}

Keywords: Barber traps, entomofauna, walnut orchard.

\section{INTRODUCTION}

In the complex of control measures to ensure the health of trees, an important role has recently returned to chemical control.

Control must be applied in accordance with the new ecological concept of integrated control, which consists of a system for regulating pest populations, taking into account the specific flight and dynamics of pest and zoophagous species, using harmoniously all methods of pest control. control (agro-phytotechnical, physical-mechanical, biological and chemical), in order to maintain the density of pests or their attack at a level that does not produce crop losses.

This integrated control system is a set of methods, means, products that are applied according to various criteria in crop technology to reduce losses.

It is necessary to take into account taxonomic studies (determination of host and parasite species), biological studies (food source, mode of attack and feeding, stages of development, duration of each stage, mode of multiplication, the number of generations), ecology studies (influence of climatic factors, establishing the relations between the attacked and harmful species, between pests and parasitic species, the importance of parasites in limiting the pest population). 
These studies offer the possibility of directed human intervention, in order to reduce damage and restore biocenotic balances in ecosystems and they will be the basis for the judicious preparation of biological and integrated pest control schemes.

\section{MATERIALS AND METHODS}

In 2019 and 2020 there were collected the entomofauna of invertebrates existing in walnut orchards. To establish biodiversity, ecological dynamics and calculation of indices such as (Talmaciu, 2005) abundance (A), constance (C) dominance (D), the index of ecological significance (W) etc. In a walnut plantation were placed the soil traps type Barber for our research for all type of arthropods. There were a number of 6 traps installed in a walnut plantation from Iasi (Butnariu, 2020). These pots consisted of approximately $800 \mathrm{ml}$ capacity which was placed a solution of salt in a $20 \%$ concentration (Herea, 2019). The traps were installed in April- May and worked until August to September, over two years.

The material who was collected were removed from plant debris, soil particles and other material, whichever is only arthropods fauna.

To follow the dynamics of species of invertebrates collected, there have been periodic harvests of traps, samples each time tagging, labeling specifying the number and date of collection trap.

In both years of observations have been made by 7 harvests of the collected material, covering in this way almost the entire growing season of trees.

\section{RESULTS AND DISCUSSIONS}

In 2019 in total, 1-6 traps were collected 255 specimens belonging to more groups of species (taxa) (Ritter,1908) (Rogojanu and Perju, 1979). Most specimens were collected from the harvest VII, 71, followed by harvesting the fifth, sixth 51 specimens and 41 specimens of the sixth harvest. The few specimens were collected from harvesting II-IV, between 17 and 26 samples.

In the 6 traps at 7 harvests there were collected the specimens belonging to a number of 22 species, the species with the highest number of specimens collected are: Dermestes laniarius 42 specimens, Heteroptera (bedbugs), with 38 specimens, Cyaniris cinerea with 29 specimens and 26 specimens Polydrosus sericeus. The lowest number of specimens, they had two species: Anisodactylus binotatus F., Balanitis glandium L., other species (taxa) that Podonta nigra F.and Silpha obscura had between 3 and 14 pieces (Tab. 1)

Referring to the number of traps that each species was collected in 2019 from the walnut plantations shows the following in table 1 . The most frequently collected species were Heteroptera in 10 traps, followed by species Dermestes laniarius L. Polydrosus sericeus, collected by 5 traps, Coccinella septempunctata into 4 traps species of Homoptera (cycads) and Tomoxia biguttata in by 3 traps, the other species were collected only in one or two traps.

For a deeper analysis of how the results were calculated a number of leading ecological indexes such as abundance (A), consistency (C) dominance (D) and the ecological significance index (W). These indicators were calculated with the results centralizated in 2019 (tab. 2) for the all colected species of arthropods.

- The Abundance largest had a species: (42 specimens), species Heteroptera (38 specimens), Cyanis cinerea (34 specimens), Polydrosus sericeus Schall (26 specimens), Coccinella septempunctata L (14 specimens), Homoptera (cycads) and Harpalus distinguendus Duft (12 specimens). The other species had between 2 and 9 specimens. 
Current Trends in Natural Sciences

Vol. 10, Issue 19, pp. 226-234, 2021

https://doi.org/10.47068/ctns.2021.v10i19.030

Current Trends in Natural Sciences (on-line)

Table 1. The species (taxons) and the number of specimens collected in 2019, from walnut orchards in Iași

\begin{tabular}{|c|c|c|c|c|c|c|c|c|c|}
\hline Nr. & Name of species (taxa) & 28.05 . & 30.05 & 15.06 & 12.07 & 26.07. & 10.08 . & 23.08. & Total \\
\hline 1. & Dermestes laniarius & - & - & - & 3 & 6 & 3 & $12+18$ & 42 \\
\hline 2. & Anisodactylus binotatus $F$. & - & - & - & 2 & - & - & - & 2 \\
\hline 3. & Polydrosus sericeus Schall & $9+3+9$ & - & 4 & - & - & - & 1 & 26 \\
\hline 4. & Coccinella septempunctata & 5 & - & 7 & $4+3$ & - & 2 & - & 14 \\
\hline 5. & Polydrosus amoenus Schall & - & - & - & - & 9 & - & - & 9 \\
\hline 6. & Tomoxia biguttata & - & 2 & - & - & 3 & - & 3 & 8 \\
\hline 7. & Chilopoda longitarsis & - & - & - & - & 2 & - & - & 7 \\
\hline 8. & Heteroptere (bedbugs) & 2 & - & $3+5$ & - & $3+2+4$ & $12+3+3$ & 1 & 38 \\
\hline 9. & Balanitis glandium L. & - & 2 & - & - & - & - & - & 2 \\
\hline 10. & Amara aenea & - & 4 & - & - & 3 & - & - & 7 \\
\hline 11. & Necrophorus vespillo $L$. & - & - & - & - & - & 3 & - & 3 \\
\hline 12. & Cyaninis cyanea $F$ & - & - & - & - & - & 10 & 19 & 34 \\
\hline 13. & Homoptere(cycads) & - & - & - & - & 6 & - & $3+3$ & 12 \\
\hline 14. & Silpha obscura & - & - & - & - & - & - & 2 & 2 \\
\hline 15. & Hymenoptera (wasp) & - & 3 & - & - & - & - & 2 & 5 \\
\hline 16. & Cymindis vaporariorum $L$. & - & - & - & - & - & 4 & 2 & 6 \\
\hline 17. & Harpalus calceatus Duft & - & - & 3 & - & - & - & - & 3 \\
\hline 18. & Podonta nigrita $F$ & - & - & 2 & - & - & - & - & 2 \\
\hline 19. & Galeruca pomonae & - & - & 2 & - & - & - & - & 2 \\
\hline 20. & Armadillidium vulgare & - & 3 & - & - & - & - & - & 3 \\
\hline 21. & Harpalus distinguendus & - & 12 & - & - & - & - & - & 12 \\
\hline 22. & Ophonus azureus & - & - & - & - & 5 & - & - & 5 \\
\hline \multicolumn{2}{|c|}{ Total 22 species } & 28 & 26 & 19 & 17 & 51 & 41 & 71 & 255 \\
\hline
\end{tabular}

- Constancy of collected species ranged between 3.7 and 35.71. The species with the highest values of constancy were Heteroptera (35.71) Polydrosus sericeus Schall and Dermestes laniarius L. (17.85), Coccinella septempunctata (14.28), Homoptera (cycads) and Tomoxia biguttata (10.71). The lowest values of constancy (3.57) have had a total of 10 species, namely: Polydrosus amoenus Schall., Ophonus azureus F., Necrophorus vespillo L, Harpalus calceatus Duft, Armadillidium vulgare L., Anisodactylus binotatus F., Balaninus glandium L., Podonta nigra F. and Galeruca pomonae $\mathrm{F}$.

- Dominance (D) had the highest values species: Dermestes laniarius (17.23), Heteroptera (15.57), Cyaniris cyanea (13.93), Polydrosus sericeus (10.65) and Coccinella septempunctata (5.73). The other species had values less than 5.00 dominance;

- Ecological significance of the index (W) had values greater than 1.00 at a number of 3 species. These were: Heteroptera - bedbugs (5.56) Dermestes laniarius L. (3.07) and Polydrosus sericeus (1.90).

The large groups of taxa species collected the situation is as follows (tab. 3 fig. 1):

-The Coleoptera species are the most numerous, accounting for $76.25 \%$ of the total followed by Heteroptera with $15.51 \%$ of the total;

-The lowest share, 5\% have had Homoptera (4.03\%), Hymenoptera (2.96\%) and Isopoda (1.25\%). 


\section{Current Trends in Natural Sciences}

Vol. 10, Issue 19, pp. 226-234, 2021

https://doi.org/10.47068/ctns.2021.v10i19.030

Current Trends in Natural Sciences (on-line)

Table 2. Values of ecological indices of species (taxons) in 2019, in the walnut orchards from Iași

\begin{tabular}{|c|c|c|c|c|c|}
\hline \multirow{2}{*}{ No. } & \multirow{2}{*}{ Name of species (taxa) } & \multicolumn{4}{|c|}{ ECOLOGICAL INDEX } \\
\hline & & $\mathbf{A}$ & $\mathrm{C}$ & D & $\mathbf{W}$ \\
\hline 1. & Dermestes laniarius & 42 & 17.85 & 17.23 & 3.07 \\
\hline 2. & Heteroptera (bedbugs) & 38 & 35.71 & 15.57 & 5.56 \\
\hline 3. & Cyaninis cyanea $\mathrm{F}$. & 34 & 7.14 & 13.93 & 0.99 \\
\hline 4. & Polydrosus sericeus Schall & 21 & 17.85 & 10.65 & 1.90 \\
\hline 5. & Coccinella septempunctata & 14 & 14.28 & 5.73 & 0.81 \\
\hline 6. & Homoptera (cycade) & 12 & 10.71 & 4.91 & 0.52 \\
\hline 7. & Polydrosus amoenus Schall & 9 & 3.57 & 3.68 & 0.13 \\
\hline 8. & Tomoxia biguttata & 8 & 10.71 & 3.28 & 0.35 \\
\hline 9. & Chilopoda longitarsis & 7 & 7.14 & 2.86 & 0.20 \\
\hline 10. & Amara aenea & 7 & 7.14 & 2.86 & 0.23 \\
\hline 11. & Harpalus distinguendus & 12 & 3.57 & 4.96 & 0.18 \\
\hline 12. & Cymindis vaporariorum $\mathrm{L}$. & 6 & 7.14 & 2.46 & 0.18 \\
\hline 13. & Hymenoptera(wasp) & 5 & 7.14 & 2.05 & 0.15 \\
\hline 14. & Ophonus azureus & 5 & 3.57 & 2.04 & 0.07 \\
\hline 15. & Necrophorus vespillo L. & 3 & 3.57 & 123 & 0.04 \\
\hline 16. & Harpalus calceatus Duft & 3 & 3.57 & 1.23 & 0.04 \\
\hline 17. & Armadillidium vulgare & 3 & 3.57 & 1.23 & 0.03 \\
\hline 18. & Anisodactylus binotatus $\mathrm{F}$. & 2 & 3.57 & 0.82 & 0.03 \\
\hline 19. & Balaninus glandium L. & 2 & 3.57 & 0.82 & 0.03 \\
\hline 20. & Harpalus distinguendus & 3 & 3.57 & 1.23 & 0.04 \\
\hline 21. & Podonta nigrita $\mathrm{F}$. & 2 & 3.57 & 0.82 & 0.03 \\
\hline 22. & Galeruca pomonae & 2 & 3.57 & 0.82 & 0.03 \\
\hline \multicolumn{2}{|c|}{ Total 22 species } & \multicolumn{4}{|c|}{255 colected samples } \\
\hline
\end{tabular}

Table 3. The structure of the collected entomofauna of walnut plantations, in 2019, groups of taxons

\begin{tabular}{||l|l|l|l||}
\hline No. & Taxa & No. of colected samples & \% total (255) \\
\hline 1. & Coleoptera & 186 & 76.25 \\
\hline 2. & Heteroptera & 38 & 15.51 \\
\hline 3. & Homoptera & 12 & 4.03 \\
\hline 4. & Hymenoptera & 5 & 2.96 \\
\hline 5. & Isopoda & 3 & 1.25 \\
\hline TOTAL & $\mathbf{5}$ taxa & $\mathbf{2 5 5}$ & 100 \\
\hline
\end{tabular}

In 2020 in total, of 6 traps were collected 756 specimens belonging to several groups of species (taxa) (Ritter 1908) (Rogojanu and Perju 1979). The most specimens were collected from the harvest III, 218, followed by harvesting fourth, 99 specimens and VII of the harvest to 97 specimens. The few specimens were collected from 72 specimens fifth harvesting.

At the 6 traps at 7 harvests were collected specimens belonging to a number of 41 species, the species with the highest number of specimens collected are: Hymenoptera 92 specimens, Orthoptera (grasshoppers) with 87 specimens, Lepidoptera (larvae) 42 specimens , Araneide, Hymenoptera (ants) with 33 specimens, Homoptera (cycads) 31 specimens, Hymenoptera (wasps) with 30 specimens Diptera (larvae) with 29 specimens, Galeruca tanaceti 25 specimens, Opatrum sabulosum 24 specimens, Diptera (adults) with 23 specimens, Coccinella conglobata 21 specimens Ontophagus ovatus and Hymenoptera (ants) with 20 specimens, Dermestes laniarius 18 specimens Ceutorhinchus crucifer and Ontophagus taurus 17 specimens, Polydrosus sericeus 16 specimens 
Gastropoda 13 specimens Tomoxia bigutatta, Polydrosus amoeus and Harpalus calceatus 12 specimens and 11 specimens Amara aenea (tab. 4)

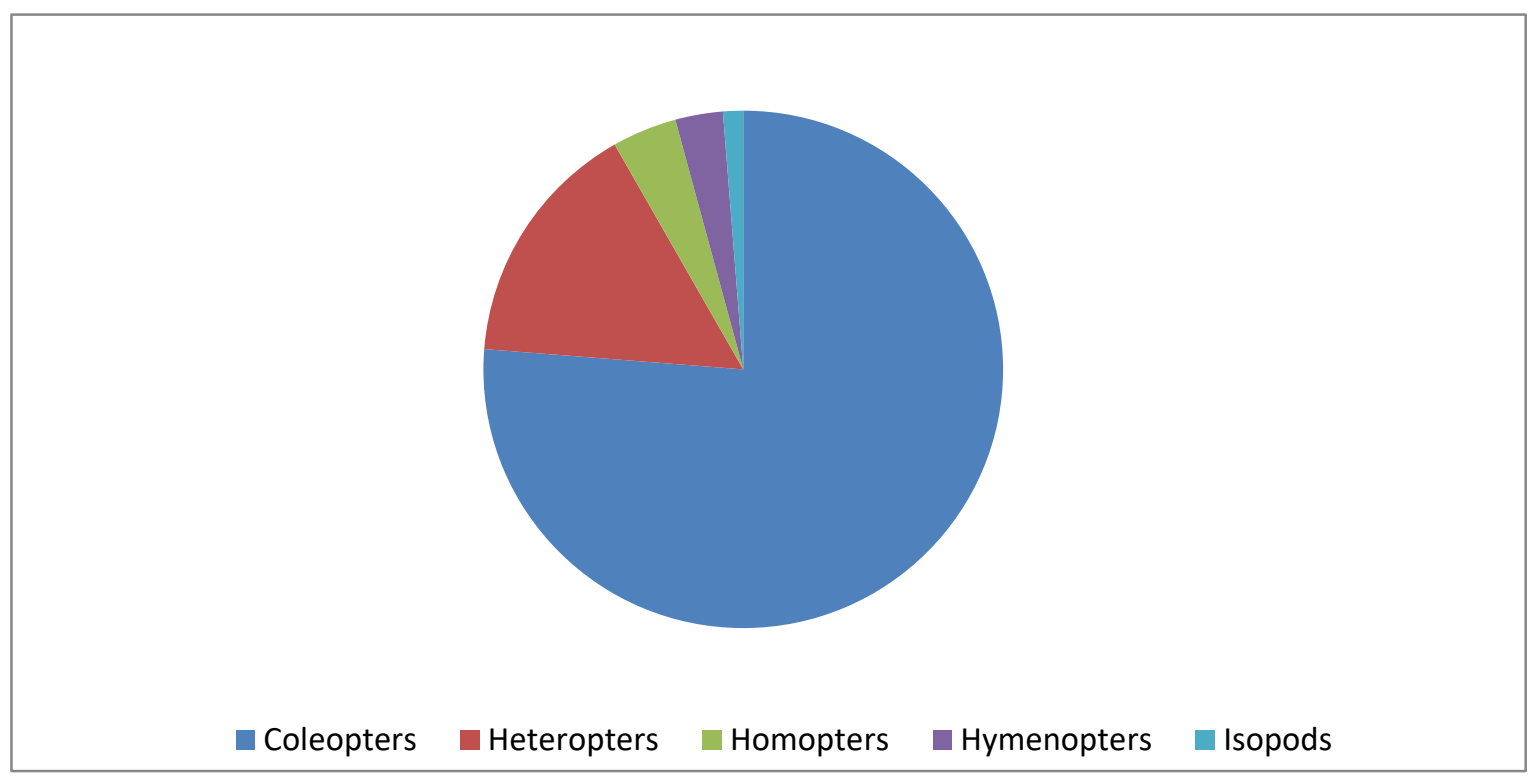

Figure 1. The structure of collected taxons from walnut plantations in 2019

Other species recorded a number equal to or less than 10 specimens.

For a deeper analysis of how the results were calculated a number of leading ecological indexes such as abundance (A), constancy (C) dominance (D) and ecological significance index (W).

These indicators was calculated in 2020 for all species or taxa collected in walnut orchards and that is as follows (tab. 5)

The Abundance most had a species: Hymenoptera (bees) (92 specimens), Orthoptera (locusts) (87 specimens), Lepidoptera (larvae) (37 specimens), Hymenoptera (wasps) (36 specimens), Araneide, Hymenoptera (ants) (33 specimens), Homoptera (cycads) (31 specimens), Opatrum sabulosum (26 specimens), Galeruca tanaceti (25 specimens), Coccinella conglobata (21 specimens), Ontophagus ovatus (20 specimens), Dermestes laniarius (18 specimens), Diptera (larvae) and Ontophagus taurus (17 specimens), Polydrosus sericeus (16 specimens), Harpalus distinguendus and Gastropoda (13 specimens) Polydrosus amoeus and Tomoxia bigutatta and Harpalus calceatus (12 specimens), Amara aenea (11 specimens) Hister purpurascens and Heteroptera (bugs)(1 specimens). The other species had between 2 and 9 specimens;

- The Constance collected species ranged between 2.70 and 37.83. The species with the highest values of constancy were Orthoptera (grasshoppers (37.83), Lepidoptera (larvae) (29.73), Hymenoptera (wasps) and Araneide (27.02), Hymenoptera (bees) (24.32), adults Diptera (21.62), Galeruca tanaceti (16.22), Opatrum sabulosum and Hymenoptera (ants) (10,81). the lowest values of constancy under (8.12) have had a number of 7 species of which: Harpalus distinguendus Duft., Gastropoda, Dermestes laniarius, Diptera (larvae) and Homoptera (cycads).

- Dominance (D) had the highest values species: Hymenoptera (bees) (12.36), Orthoptera (11.39), Lepidoptera (adults) (11.21), and other species showed values between 0-14 and 4.43. 


\section{Current Trends in Natural Sciences}

Vol. 10, Issue 19, pp. 226-234, 2021

https://doi.org/10.47068/ctns.2021.v10i19.030

Current Trends in Natural Sciences (on-line)

ISSN: 2284-953X

Current Trends in Natural Sciences (CD-Rom)

ISSN: 2284-9521

ISSN-L: 2284-9521

ISSN-L: 2284-9521

- Index of ecological significance (W) had higher values of 1.00 to a total of five species. These were: Orthoptera (locusts) (4.42), Hymenoptera (bees) (3), Lepidoptera (larvae) (1.48) Araneide (1.19), Hymenoptera (wasps) (1.08)

Table 4. The species (taxons) and the number of samples collected in 2020

\begin{tabular}{|c|c|c|c|c|c|c|c|c|c|}
\hline No & Name of species (taxa) & 07.05 & 21.05 & 07.07 & 04.08 & 04.09 & 12.09 & 27.09 & Total \\
\hline 1. & Pterostichus niger Sch & 3 & - & - & - & - & - & - & 3 \\
\hline 2. & Araneida & $2+8$ & - & $3+5+2+2$ & 3 & - & $3+2$ & 3 & 33 \\
\hline 3. & Diptera (adults) & $1+6+1$ & $2+2$ & 5 & 3 & - & - & 3 & 23 \\
\hline 4. & Opatrum sabulosum & $8+6$ & - & - & - & - & - & $6+6$ & 26 \\
\hline 5. & Hymenoptera (wasp) & $6+2$ & & 1 & $6+3+6$ & 3 & - & 3 & 30 \\
\hline 6. & Harpalus distinguendus & 8 & - & - & - & - & - & - & 8 \\
\hline 7. & Gastropoda & - & 2 & - & - & 6 & - & 5 & 13 \\
\hline 8. & Tomoxia biguttata & - & 6 & 6 & - & - & - & - & 12 \\
\hline 9. & Hister purpurascens & - & - & 5 & - & 5 & - & - & 10 \\
\hline 10. & Hymenoptera (bees) & 2 & 5 & $20+10+21+8+9$ & - & $6+3+10$ & - & - & 92 \\
\hline 11. & Galeruca tanaceti & 3 & - & 4 & 6 & 3 & $3+3$ & 6 & 25 \\
\hline 12. & Harpalus calceatus & - & - & $6+6$ & - & - & - & - & 12 \\
\hline 13. & Lepidoptera (larva) & $1+5$ & $4+8+3$ & 5 & $1+3+2+2$ & - & - & 3 & 37 \\
\hline 14. & Dermestes laniarius & - & - & - & 3 & 6 & - & 9 & 18 \\
\hline 15. & Hymenoptera (ants) & - & 20 & 8 & 5 & - & - & - & 33 \\
\hline 16. & Armadillidium vulgare & - & - & - & 3 & - & - & - & 3 \\
\hline 17. & Orthoptera (locust) & - & $9+6$ & $2+2$ & - & $3+3+12$ & $9+26$ & $6+6+3$ & 87 \\
\hline 18. & Harpalus distinguendus & - & - & - & - & 5 & - & - & 5 \\
\hline 19. & Diptera (larva) & $1+2$ & - & 4 & 12 & - & - & 10 & 29 \\
\hline 20. & Ceutorhynchus crucifer & 2 & 4 & $6+5$ & - & - & - & - & 17 \\
\hline 21. & Blaps lethifera & - & - & 3 & - & - & - & - & 3 \\
\hline 22. & Orthoptera (Gryllus) & 3 & - & - & - & 3 & - & - & 6 \\
\hline 23. & Onthophagus taurus & - & - & - & - & - & $15+2$ & - & 17 \\
\hline 24. & Cantharis fusca. & 3 & - & 5 & - & - & - & - & 3 \\
\hline 25. & Polydrosus sericeus & 3 & - & - & 9 & - & 4 & - & 16 \\
\hline 26. & Homoptera (cycads) & - & 6 & 13 & - & - & 12 & - & 31 \\
\hline 27. & Carabus scabriusculus & - & 5 & - & - & - & - & - & 5 \\
\hline 28. & Leipidoptere (adults) & - & - & $3+3$ & - & - & 3 & - & 9 \\
\hline 29. & Amara aenea & - & 2 & 9 & - & - & - & - & 11 \\
\hline 30. & Pseudophonus griseus & - & - & 8 & - & - & - & - & 8 \\
\hline 31. & Coccinella conglobata & - & - & - & $12+9$ & - & - & - & 21 \\
\hline 32. & Anisodactylus binotatus & - & - & - & - & 5 & - & 4 & 4 \\
\hline 33. & Calathus fuscipes Gaeze & 4 & - & - & - & - & - & - & 4 \\
\hline 34. & Hymenoptera (ants) & - & 20 & - & - & - & - & - & 20 \\
\hline 35. & Polydrosus amoeus & - & - & 5 & $6+6$ & - & - & - & 12 \\
\hline 36. & Carabus coriaceus & - & - & - & - & - & $3+3$ & - & 6 \\
\hline 37. & Onthophagus ovatus & - & - & 5 & - & - & - & $6+9$ & 20 \\
\hline 38. & Homoptera (aphids) & 8 & - & - & - & - & - & - & 8 \\
\hline 39. & Collembole & 2 & - & - & - & - & - & - & 2 \\
\hline 40. & Adalia bipunctata & - & - & - & 3 & - & - & - & 3 \\
\hline 41. & Heteroptera (bedbug) & - & - & 10 & - & - & - & - & 10 \\
\hline \multicolumn{2}{|c|}{ Total 41 species } & 90 & 90 & 218 & 99 & 72 & 90 & 97 & 756 \\
\hline
\end{tabular}




\section{Current Trends in Natural Sciences}

Vol. 10, Issue 19, pp. 226-234, 2021

https://doi.org/10.47068/ctns.2021.v10i19.030

Current Trends in Natural Sciences (on-line)

The large groups of taxa species collected the situation is as follows (tab. 6, fig. 2):

- Beetles are the most numerous, accounting for $38.70 \%$ of the total followed by Hymenoptera with $23.52 \%$ of the total;

- $\quad$ The lowest share, 5\% have had Araneide (4.43\%), Gastropoda (1.74\%) and Collembola $(0.27 \%)$.

Table 5. The values of ecological indices for species (taxons) in 2020, in the walnut orchard

\begin{tabular}{|c|c|c|c|c|c|}
\hline \multirow{2}{*}{ NO. } & \multirow{2}{*}{ Name of species (taxa) } & \multicolumn{4}{|c|}{ ECOLOGIC INDEX } \\
\hline & & $\mathbf{A}$ & $\mathbf{C}$ & $\mathbf{D}$ & $\mathbf{W}$ \\
\hline 1. & Pterostichus niger Schall & 3 & 2.70 & 0.40 & 0.01 \\
\hline 2. & Araneida & 33 & 27.02 & 4.43 & 1.19 \\
\hline 3. & Diptera (adults) & 23 & 21.62 & 3.09 & 0.67 \\
\hline 4. & Opatrum sabulosum & 26 & 10.81 & 3.49 & 0.38 \\
\hline 5. & Hymenoptera (wasp) & 36 & 27.02 & 4.03 & 1.08 \\
\hline 6. & Harpalus distinguendus & 13 & 8.11 & 1.75 & 0.14 \\
\hline 7. & Gastropoda & 13 & 8.11 & 1.75 & 0.14 \\
\hline 8. & Tomoxia biguttata & 12 & 5.41 & 1.61 & 0.09 \\
\hline 9. & Hister purpurascens & 10 & 5.41 & 1.34 & 0.07 \\
\hline 10. & Hymenoptera (beens) & 92 & 24.32 & 12.36 & 3.00 \\
\hline 11. & Galeruca tanaceti & 25 & 16.22 & 3.36 & 0.54 \\
\hline 12. & Harpalus calceatus & 12 & 5.41 & 1.61 & 0.09 \\
\hline 13. & Lepidoptera (larva) & 37 & 29.73 & 4.97 & 1.48 \\
\hline 14. & Dermestes laniarius & 18 & 8.11 & 2.41 & 0.20 \\
\hline 15. & Hymenoptera (ants) & 33 & 10.81 & 4.43 & 0.48 \\
\hline 16. & Armadillidium vulgare & 3 & 2.70 & 0.40 & 0.01 \\
\hline 17. & Orthoptera (locust) & 87 & 37.83 & 11.69 & 4.42 \\
\hline 18. & Diptera (larva) & 17 & 8.11 & 2.28 & 0.18 \\
\hline 19. & Ceutorhynchus crucifer & 3 & 2.70 & 0.40 & 0.01 \\
\hline 20. & Blaps lethifera & 6 & 5.40 & 0.80 & 0.04 \\
\hline 21. & Orthoptera (Gryllus) & 3 & 2.70 & 0.40 & 0.01 \\
\hline 22. & Onthophagus taurus & 17 & 5.40 & 2.28 & 0.121 \\
\hline 23. & Polydrosus sericeus Schall & 16 & 8.11 & 2.15 & 0.17 \\
\hline 24. & Homoptera (cycads) & 31 & 8.11 & 4.17 & 0.34 \\
\hline 25. & Carabus scabriusculus & 5 & 2.70 & 0.67 & 0.02 \\
\hline 26. & Leipidoptere (adults) & 9 & 8.1 & 11.21 & 0.09 \\
\hline 27. & Amara aenea & 11 & 5.40 & 1.48 & 0.08 \\
\hline 28. & Pseudophonus griseus & 8 & 2.70 & 1.08 & 0.03 \\
\hline 29. & Coccinella conglobata & 21 & 5.40 & 2.82 & 0.15 \\
\hline 30. & Anisodactylus binotatus $F$ & 7 & 5.40 & 0.14 & 0.05 \\
\hline 31. & Calathus fuscipes Gaeze & 4 & 2.70 & 0.54 & 0.01 \\
\hline 32. & Polydrosus amoeus & 12 & 5.40 & 1.61 & 0.08 \\
\hline 33. & Carabus coriaceus $L$ & 6 & 5.40 & 0.81 & 0.04 \\
\hline 34. & Onthophagus ovatus. & 20 & 8.11 & 2.68 & 0.22 \\
\hline 35. & Homoptera (aphyds) & 8 & 2.70 & 1.08 & 0.03 \\
\hline 36. & Collembole & 2 & 2.70 & 0.40 & 0.01 \\
\hline 37. & Adalia bipunctata & 3 & 2.70 & 0.40 & 0.01 \\
\hline & Heteroptera (bedbugs) & 10 & 2.70 & 1.34 & 0.04 \\
\hline \multicolumn{2}{|c|}{ Total 38 species } & & \multicolumn{3}{|c|}{756 samples colected } \\
\hline
\end{tabular}


Current Trends in Natural Sciences

Vol. 10, Issue 19, pp. 226-234, 2021

https://doi.org/10.47068/ctns.2021.v10i19.030

Current Trends in Natural Sciences (on-line)

Table 6. The structure entomofauna collected of walnut plantations, in 2020

\begin{tabular}{|l|l|c|c|}
\hline No.. & Name of species (taxa) & No. of specimens & \% total (744) \\
\hline 1. & Araneida & 33 & 4.3 \\
\hline 2. & Diptera & 52 & 6.98 \\
\hline 3. & Hymenoptera & 175 & 23.52 \\
\hline 4. & Lepidoptera & 46 & 6.18 \\
\hline 5. & Izopode & 3 & 0.40 \\
\hline 6. & Gastropoda & 13 & 1.74 \\
\hline 7. & Orthoptera & 93 & 12.50 \\
\hline 8. & Homoptera & 39 & 5.24 \\
\hline 9. & Collembole & 2 & 0.27 \\
\hline 10. & Coleoptere & 288 & 38.70 \\
\hline
\end{tabular}

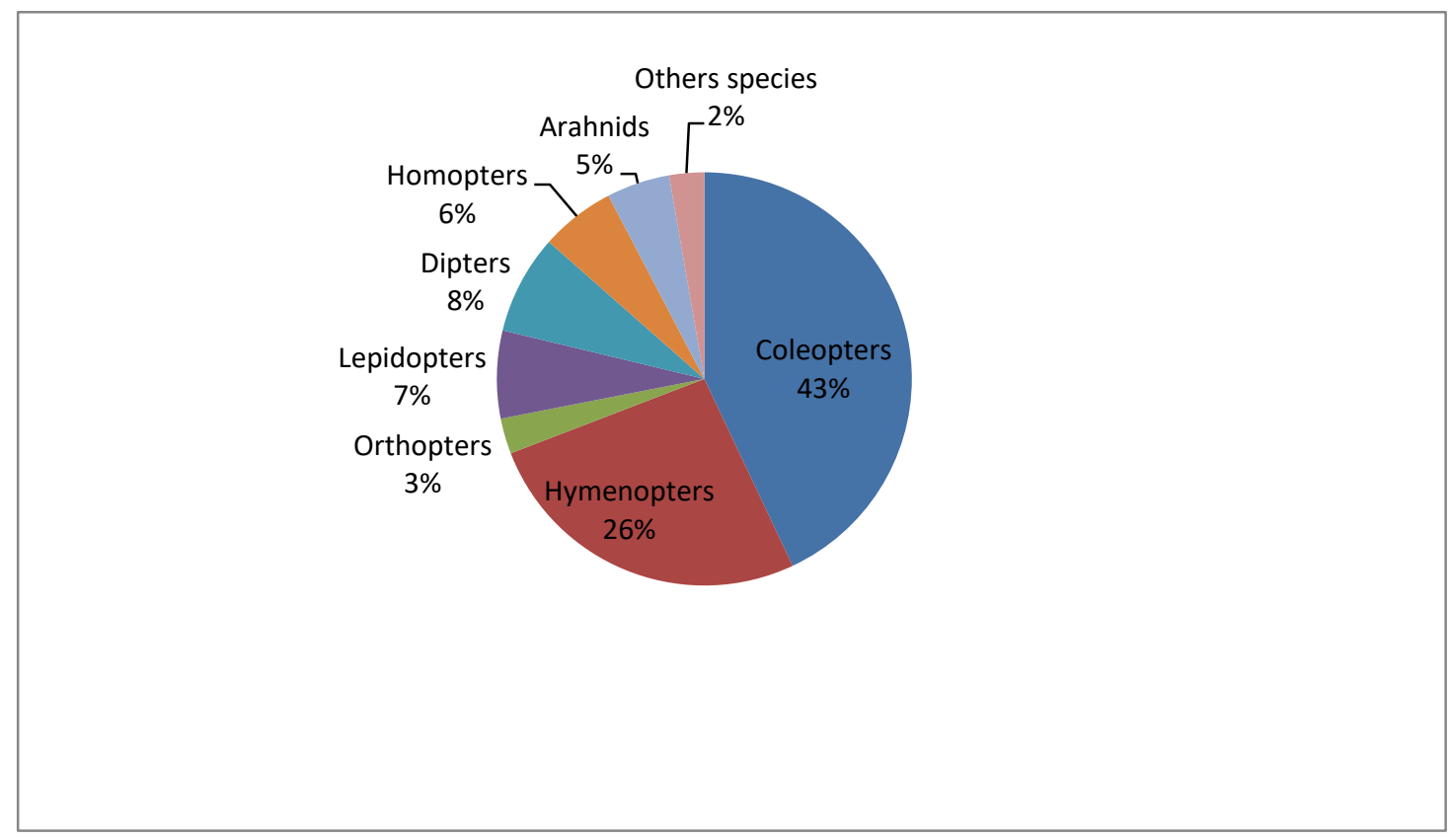

Figure 2. The structure of collected taxons from walnut plantations in 2020

\section{CONCLUSIONS}

1. 2019 was collected in the orchards of walnut specimens belonging to more and different groups of taxa as follows:

- Beetles (Coleoptera), 186 specimens representing $76.25 \%$ of the total (255 copies);

- Heteroptera, 38 specimens representing $15.51 \%$ of the total;

- Homoptera 12 specimens reprezentâmnd $4.03 \%$ of the total;

- Hymenoptera 5specimens, representing $2.96 \%$ of the total;

- Isopoda, 3 specimens, representing $1.25 \%$ of the total.

2. In 2020, were collected from orchards of walnut samples of taxa belonging to the following groups:

- Beetles (Coleoptera), representing 38288 specimens, $70 \%$ of the total (756 specimens); 
Current Trends in Natural Sciences

Vol. 10, Issue 19, pp. 226-234, 2021

https://doi.org/10.47068/ctns.2021.v10i19.030

Current Trends in Natural Sciences (on-line)

ISSN: 2284-953X

Current Trends in Natural Sciences (CD-Rom)

ISSN: 2284-9521

ISSN-L: 2284-9521

ISSN-L: 2284-9521

- Hymenoptera 175 specimens, representing $23.52 \%$ of the total;

- Orthoptera, 93 specimens representing $12.50 \%$ of the total

- Diptera, 52 specimens representing $6.98 \%$ of the total;

- Lepidoptera, 46 specimens representing $6.18 \%$ of the total;

- Homoptera, 39specimens reprezentâmnd 5.24\% of the total;

- Araneide, 33 specimens representing $4.43 \%$ of the total;

- Gastropoda, 13 specimens representing $1.74 \%$ of the total;

- Isopoda, 3 specimens, representing $0.40 \%$ of the total.

- Collembola, 2 specimens, representing $0.27 \%$ of the total.

\section{REFERENCES}

Butnariu, G., et al. (2014) Preliminary studies on the main pest control methods of plum plantations. Lucrări științifice, seria Agronomie, 57- 2, 163-166.

Herea, M., Talmaciu, M., Boboc, C., Talmaciu, N. (2019) Observations on existing arthropods fauna in some orchards, Lucrări Ştiinţifice Seria Horticultură, USAMV Iaşi, 62 (2), 87-92.

Reitter, E. (1908) Fauna Germanica. Die Käfer des Deutschen Reiches Band I, Stuttgart.

Rogojanu, V., Perju, T. (1979) Determinant for the pests recognition of agricultural plants. Editura Ceres, Bucureşti.

Talmaciu, M. (2005) The structure, abundance and dynamics of the coleoptere species from the vineyard in the Huşi viticultural ecosystem, Vaslui Department.. Lucrări ştiinţifice, Chişinău, 13, 146-155. 